\title{
ON THE SUPPORT VARIETIES FOR DEMAZURE MODULES
}

\author{
BENJAMIN F. JONES and DANIEL K. NAKANO ${ }^{\bowtie}$
}

(Received 30 August 2010; accepted 11 November 2011)

Communicated by J. Du

\begin{abstract}
The support varieties for the induced modules or Weyl modules for a reductive algebraic group $G$ were computed over the first Frobenius kernel $G_{1}$ by Nakano, Parshall and Vella. A natural generalization of this computation is the calculation of the support varieties of Demazure modules over the first Frobenius kernel, $B_{1}$, of the Borel subgroup $B$. In this paper we initiate the study of such computations. We complete the entire picture for reductive groups with underlying root systems $A_{1}$ and $A_{2}$. Moreover, we give complete answers for Demazure modules corresponding to a particular (standard) element in the Weyl group, and provide results relating support varieties between different Demazure modules which depend on the Bruhat order.
\end{abstract}

2010 Mathematics subject classification: primary 17B56; secondary 17B10, $20 \mathrm{G} 10$.

Keywords and phrases: support varieties, cohomology, Demazure modules.

\section{Introduction}

Let $G$ be a connected, simply connected, simple algebraic group scheme defined over $\mathbb{F}_{p}$. Moreover, let $W$ be the associated Weyl group, $B$ be a Borel subgroup and $X(T)_{+}$ be the set of dominant weights. Given $w \in W$ and $\lambda \in X(T)_{+}$, the Demazure module, labeled by $H^{0}(w, \lambda)$, is constructed using iterated inductions involving parabolics corresponding to simple reflections occurring in a reduced decomposition of $w$. When $w=w_{0}$ is the long element of $W$, one recovers the induced $G$-modules $H^{0}(\lambda)=\operatorname{ind}_{B}^{G} \lambda$, which can be realized as global sections of the line bundle $\mathcal{L}(\lambda)$ over $G / B$.

Demazure modules arise naturally as the global sections on a line bundle $\mathcal{L}(\lambda)$ on the Schubert scheme $X(w)$ [12, Ch. 14]. The structure of Demazure modules, and $B$-modules with excellent filtration in general, is closely related to the geometry of the underlying Schubert varieties (resolution of singularities, sheaf cohomology, normality and rational singularities). For example, Mehta and Ramanathan, using the technique of Frobenius splittings, and later Andersen, using representation-theoretic

The first-named author was supported in part by NSF VIGRE grant DMS-0738586, and the second-named author was supported in part by NSF VIGRE grant DMS-1002135.

(C) 2012 Australian Mathematical Publishing Association Inc. 1446-7887/2012 \$16.00 
techniques, showed that the analog of Kempf's vanishing theorem holds for sections of a dominant line bundle restricted to a Schubert variety. This result was applied to complete Demazure's proof of his character formula. As another example, Polo [15] and van der Kallen [19] used the normality of Schubert varieties in a crucial way in their investigation of the category of $B$-modules with excellent filtration.

In 2002, at a workshop in Seoul, Korea, B. Parshall proposed the problem of computing the support varieties of the Demazure modules $H^{0}(w, \lambda)$ over the first Frobenius kernel $B_{1}$. This problem is a natural and interesting extension of the 'Jantzen conjecture' on support varieties, which predicted the support varieties of $H^{0}(\lambda)$ over $G_{1}$ when the characteristic of the field is good. The conjecture was verified by Nakano et al. [14] and the support varieties of $H^{0}(\lambda)$ over $G_{1}$ were shown to be closures of Richardson orbits. This computation was later extended to fields of bad characteristic by the University of Georgia VIGRE Algebra Group [18]. In the latter case, the support variety of $H^{0}(\lambda)$ is still irreducible and is the closure of an orbit, but the orbits need not be Richardson.

Support varieties are natural with respect to the inclusion of $B_{1}$ in $G_{1}$, so one can deduce from the aforementioned results that the $B_{1}$ support varieties of $H^{0}(\lambda)$ will be unions of closures of orbital varieties (see [13]). The orbital varieties arise as the irreducible components in the intersections of $G$-orbits on the nilpotent cone with the nilradical of a Borel subalgebra. Indeed, the closures of orbital varieties should play an important role in the general theory of support varieties of Demazure modules. This will be more evident in the results in this paper.

The main obstacle in computing support varieties for general Demazure modules is that these modules are rarely $G$-modules (that is, their support varieties are not $G$ invariant, and not closures of finitely many $G$-orbits). In general there are infinitely many $B$-orbits on the nilpotent radical of Lie $B$. At present it is not known how to classify these $B$-orbits. The aim of the paper is to study the behavior of support varieties of Demazure modules. In many instances we will be able to provide an explicit description of the supports.

The paper is organized as follows. In Section 2, we present various properties of Schubert varieties that will be used throughout the paper. We then discuss properties of support varieties over the Frobenius kernels $B_{r}$ and $P_{r}$. Several of the main results in [8] and [14] need to be modified and generalized for the purposes of this paper (see Theorems 3.2.1 and 3.3.1). In Section 4, we prove a $G$-saturation result for the $B_{r}$ support varieties of Demazure modules. In particular, we show that if $w_{1}<w_{2}$ (in the Bruhat order), then $G \cdot \mathcal{V}_{B_{r}}\left(H^{0}\left(w_{2}, \lambda\right)\right) \subseteq G \cdot \mathcal{V}_{B_{r}}\left(H^{0}\left(w_{1}, \lambda\right)\right)$. This result is subtle and we indicate by example that this inclusion does not hold if one ignores the process of $G$-saturation (see Example 4.1.2). With these results, we describe the supports of the Demazure modules in the $A_{1}$ case. Calculations of support varieties $\mathcal{V}_{B_{1}}\left(H^{0}(w, \lambda)\right)$ are given for specific $w \in W$ in Section 5. Finally, in Section 6, we provide a complete description of $\mathcal{V}_{B_{1}}\left(H^{0}(w, \lambda)\right)$ for algebraic groups of type $A_{2}$. An interesting facet of the $A_{2}$-computation is the need to analyze and use information about higher sheaf cohomology groups. 


\section{Schubert schemes}

We first introduce some notation. Throughout this paper, let $k$ be an algebraically closed field of characteristic $p>0$. For an algebraic group $H$, the notation $\operatorname{Mod}(H)$ denotes the category of rational $H$-modules and $\bmod (H)$ denotes the category of finitedimensional, rational $H$-modules.

Let $\Phi$ be a finite irreducible root system for a Euclidean space $\mathbb{E}$. The inner product on $\mathbb{E}$ will be denoted by $(\cdot, \cdot)$. For $\alpha \in \Phi$, let $\alpha^{\vee}=2 \alpha /(\alpha, \alpha)$ be the corresponding coroot. Fix a set $\Delta=\left\{\alpha_{1}, \ldots, \alpha_{\ell}\right\}$ of simple roots, and let $\Phi^{+}$be the corresponding set of positive roots. The Weyl group $W \subset O(\mathbb{E})$ is the group generated by the reflections $s_{\alpha}: \mathbb{E} \rightarrow \mathbb{E}, \alpha \in \Phi$, given by $s_{\alpha}(x)=x-2\left(x, \alpha^{\vee}\right) \alpha$.

Unless otherwise stated, $G$ will denote a reductive algebraic group over $k$. We will always assume that the derived group $G^{\prime}$ is simply connected. Also, assume that $G$ has root system $\Phi$ with respect to a maximal split torus $T$. Let $B \supset T$ be the Borel subgroup defined by $-\Phi^{+}$. The positive Borel subgroup containing $T$ will be denoted $B^{+}$. Moreover, let $X(T)=X(B)$ be the group of integral characters of $T$ or, equivalently, of $B$. Given $\lambda \in X(T)$, we will let $\lambda$ also denote the one-dimensional $B$-module defined by regarding $\lambda$ as a character on $B$. Then the set of dominant integral weights is defined by

$$
X_{+}:=X(T)_{+}=\left\{\lambda \in X(T): 0 \leq\left(\lambda, \alpha_{i}^{\vee}\right), 1 \leq i \leq \ell\right\} .
$$

Let $\rho$ be the half sum of the positive roots. We partially order $X(T)$ by setting $\lambda \geq \mu$ if and only if $\lambda-\mu \in \sum_{\alpha \in \Delta} \mathbb{N} \alpha$. Let $h$ be the Coxeter number of $G$. Thus, if $G^{\prime}$ is simple, $h=\left(\rho, \alpha_{0}^{\vee}\right)+1$, where $\alpha_{0}$ is the maximal short root in $\Phi$; otherwise, $h$ is the maximum of the Coxeter numbers for the simple factors of $G^{\prime}$.

Each subset $J \subset \Delta$ gives rise to a standard parabolic subgroup $P=P_{J}$ containing $B$, whose Lie algebra is generated by $\mathrm{t}=$ Lie $T$, the negative root spaces $\mathfrak{g}_{-\alpha}\left(\alpha \in \Phi_{+}\right)$and the positive root spaces in the span of $J: \mathfrak{g}_{\alpha}$ for $\alpha \in \Phi_{J}$. The subgroup $P_{J}$ has a Levi decomposition $P_{J}=L_{J} U_{J}$, where $\mathfrak{l}_{J}=$ Lie $L_{J}$ is generated by $\mathrm{t}$ and the root spaces $\mathfrak{g}_{ \pm \alpha}$ for $\alpha \in J$, and $\mathfrak{u}_{J}=\operatorname{Lie} U_{J}$ is generated by the root spaces $\mathfrak{g}_{-\alpha}$ for $\alpha \in \Phi_{+} \backslash \Phi_{J}$. We denote by $W_{J}$ the subgroup of $W$ generated by reflections $s_{\alpha}$ for $\alpha \in J$ and identify it with the Weyl group of $L_{J}$. We denote the set of minimal length right coset representatives for $W / W_{J}$ by $W^{J}$. When $P=P_{J}$, we write $W_{P}$ and $W^{P}$ rather than $W_{P_{J}}$ and $W^{P_{J}}$. We denote the opposite parabolic subgroup that contains $B^{+}$by $P_{J}^{+}$.

For $G$ as given above, the dominant weights $\lambda \in X(T)_{+}$index the simple modules $L(\lambda)$ by their highest weight. If $\operatorname{ind}_{B}^{G}: \bmod (B) \rightarrow \bmod (G)$ is the induction functor, let $H^{0}(\lambda)=\operatorname{ind}_{B}^{G} \lambda$ for $\lambda \in X(T)$. If $\lambda \notin X(T)_{+}$, then $H^{0}(\lambda)=0$, while, if $\lambda \in X(T)_{+}$, then $H^{0}(\lambda)$ has socle $L(\lambda)$.

Let $F: G \rightarrow G$ be the Frobenius morphism on $G$ induced by its $\mathbb{F}_{p}$-structure. For $r \geq 1$, put $G_{r}=\operatorname{ker}\left(F^{r}\right)$. Similarly, if $H$ is an $F$-stable subgroup of $G$, write $H_{r}=\operatorname{ker}\left(\left.F^{r}\right|_{H}\right)$; for example, $B_{r}=\operatorname{ker}\left(\left.F^{r}\right|_{B}\right)$. The group scheme $H_{r}$ is a finite $k$-group, that is, an affine algebraic group scheme over $k$ with finite-dimensional coordinate algebra $k\left[H_{r}\right]$. Also, its height is at most $r$. In what follows, all affine $k$-groups $A$ will, by definition, be assumed to be algebraic, that is, the coordinate algebra $k[A]$ is 
assumed to be finitely generated over $k$. If $M \in \operatorname{Mod}(H)$, let $M^{(r)}$ be the module in $\operatorname{Mod}(H)$ obtained by composing the representation corresponding to $M$ with $F^{r}$.

Next we follow the notation and conventions of [12, II. Chs. 13-14]. Fix a parabolic subgroup $P$. The group $G$ has a Bruhat decomposition:

$$
G=\bigcup_{w \in W^{P}} B \dot{w} P,
$$

where $\dot{w}$ denotes a chosen representative of $w$ in $N_{G}(T)$. This induces a decomposition $G / P=\bigcup B \dot{w} P / P$ into $B$-stable affine subschemes (cells). We denote by $X(w)_{P}$ the closure of the cell $B \dot{w} P / P$ in $G / P$. These are the Schubert varieties of $G / P$. When $P=B$ is a Borel subgroup, we simply use the notation $X(w)=X(w)_{B}$.

Let $M \in \bmod (P)$. The variety $G \times_{P} M$ is naturally a vector bundle over $G / P$. We denote this vector bundle by $\mathcal{L}(M)$. The most important case is when $P=B$ and $M=k_{\lambda}$ for $\lambda \in X(T)$, in which case $\mathcal{L}(M)$ is a line bundle on $G / B$. If $J \subset \Delta$ and $\lambda$ satisfies $\left(\lambda, \alpha^{\vee}\right)=0$ for all $\alpha \in J$, then there is a line bundle $\mathcal{L}(\lambda)_{P}$ on $G / P$, where $P=P_{J}$. This bundle pulls back to $\mathcal{L}(\lambda)$ on $G / B$ under the quotient map $G / B \rightarrow G / P$, which is locally trivial. Therefore, by [12, Section I 5.17], there is a canonical isomorphism $H^{0}(G / B, \mathcal{L}(\lambda)) \cong H^{0}\left(G / P, \mathcal{L}(\lambda)_{P}\right)$.

The cohomology groups $H^{i}(G / B, \mathcal{L}(M))$ are naturally $G$-modules. For each $y \in W^{P}$, the inclusion $X(y)_{P} \hookrightarrow G / P$ induces the restriction map $H^{i}(G / P, \mathcal{L}(M)) \rightarrow$ $H^{i}\left(X(y)_{P}, \mathcal{L}(M)\right)$.

The schemes $X(y)_{P}$ admit resolutions of singularities $\phi: X \rightarrow X(y)_{P}$, which are equivariant with respect to $B$ and depend on a reduced decomposition of $\dot{y}$, a minimal length coset representative of $y$ in $W$ (see [12, Section II 13.6]). The resolution $X$ is defined as a subset of a variety $Z$ that is a fiber bundle over $G / B$.

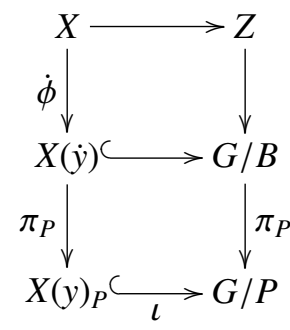

In the diagram, $\pi_{P}$ is the natural projection $G / B \rightarrow G / P$, which is birational when restricted to $X(\dot{y})$, and the resolution $\phi$ is $\phi=\pi_{P} \circ \dot{\phi}$. We need the following wellknown geometric results on Schubert varieties and sheaf cohomology.

Proposition 2.1 [12, Section II 14.15]. Let $y \in W^{P}$, let $\dot{y}$ be a minimal length right coset representative of $y$ in $W$ and let $w \in W$. Then the following hold.

(1) $X(y)_{P}$ is a normal, closed subscheme of $G / P$.

(2) For every vector bundle $V$ on $G / P$ and $i \geq 0, H^{i}\left(X(y)_{P}, V\right) \cong H^{i}\left(X(\dot{y}), \pi_{P}^{*} V\right) \cong$ $H^{i}\left(X, \phi^{*} V\right)$. 
(3) For all $\lambda \in X(T)_{+}, H^{i}(X(w), \mathcal{L}(\lambda))=0$ for $i>0$.

(4) Given $\lambda \in X(T)_{+}$such that $\left(\lambda, \alpha^{\vee}\right)=0$ for all $\alpha \in J$, where $J \subseteq \Delta$, the restriction map $H^{i}\left(G / P, \mathcal{L}(\lambda)_{P}\right) \rightarrow H^{i}\left(X(y)_{P}, \mathcal{L}(\lambda)_{P}\right)$ is surjective and moreover

$$
H^{i}\left(X(y)_{P}, \mathcal{L}(\lambda)_{P}\right)=0
$$

for all $i>0$, where $P=P_{J}$ is the standard parabolic subgroup associated to $J$.

We also need the identification of the $G$-module $H^{i}\left(G / P, \mathcal{L}(M)_{P}\right)$ with induction from $P$ to $G$.

\section{Proposition 2.2 [12, Section I 5.12].}

(1) For any $P$-module $M$ and $i \geq 0$, there is a canonical isomorphism

$$
R^{i} \operatorname{ind}_{P}^{G} M \cong H^{i}(G / P, \mathcal{L}(M)) .
$$

(2) Let $H \subset K$ be $k$-group schemes such that $K / H$ is noetherian (for example, $K$ is reductive and $H$ is a parabolic, or $H \subset K \subset G$ are both parabolic in a reductive group) and let $M$ be a rational H-module. Then

$$
R^{i} \operatorname{ind}_{H}^{K} M=0
$$

for $i>\operatorname{dim} K / H$.

\section{Support varieties over $\boldsymbol{P}_{\boldsymbol{r}}$}

In this section, let $A$ be an arbitrary finite $k$-group scheme and $\bmod (A)$ be the category of finite-dimensional $A$-modules. We will consider maximal ideals in the commutative part of the cohomology ring, so set

$$
R:=\mathrm{H}(A, k)= \begin{cases}\mathrm{H}^{2 \bullet}(A, k) & \text { if char } k \neq 2, \\ \mathrm{H}^{\bullet}(A, k) & \text { if char } k=2 .\end{cases}
$$

Friedlander and Suslin [10] proved that $R$ is a finitely generated $k$-algebra [10]. Let $\mathcal{V}_{A}$ denote the variety associated to the maximum ideal spectrum of $R$. Given $M, M^{\prime} \in$ $\bmod (A)$, we define the relative support variety $\mathcal{V}_{A}\left(M, M^{\prime}\right)=\operatorname{Maxspec}\left(R / J_{M, M^{\prime}}\right)$, where $J_{M, M^{\prime}}$ is the annihilator of the action of $R$ on $\operatorname{Ext}_{A}^{\bullet}\left(M, M^{\prime}\right)$. The action (Yoneda product) of $R=\operatorname{Ext}_{A}^{\bullet}(k, k)$ on $\operatorname{Ext}_{A}^{\bullet}\left(M, M^{\prime}\right)$ is given by taking an extension in $R$, applying $-\otimes_{k} M^{\prime}$ and then concatenating the new class with an extension class in $\operatorname{Ext}_{A}^{\bullet}\left(M, M^{\prime}\right)$ (see [3, Section 2.6]).

The ordinary support variety of $M \in \bmod (A)$ is $\mathcal{V}_{A}(M):=\mathcal{V}_{A}(M, M)$. In general, $\mathcal{V}_{A}\left(M, M^{\prime}\right)$ is a homogeneous closed subvariety contained in $\mathcal{V}_{A}=\mathcal{V}_{A}(k)$ for any $M, M^{\prime} \in \bmod (A)$. For the basic properties of support varieties for finite $k$-group schemes, we refer the reader to [9, Section 5] and [14, Section 2.2].

Let $H$ be a closed subgroup of a finite $k$-group $A$ of height at most $r$. Suslin $e t$ al. $[17,(5.4)]$ proved that the image of the restriction map res : $\mathrm{H}(A, k)_{\text {red }} \rightarrow \mathrm{H}(H, k)_{\text {red }}$ 
contains all $p^{r}$ th powers $x^{p^{r}}$ of elements $x \in H(H, k)_{\text {red }}$, and the induced morphism res* $: \mathcal{V}_{H} \rightarrow \mathcal{V}_{A}$ maps $\mathcal{V}_{H}$ homeomorphically onto its image as a closed subvariety of $\mathcal{V}_{A}$. In this paper, we will identify the image of $\mathcal{V}_{H}$ with res* $\left(\mathcal{V}_{H}\right)$ in $\mathcal{V}_{A}$. Under this map, we have the following naturality property.

Proposition 3.1. Let $H$ be a closed subgroup of $A$. Then $\mathcal{V}_{H}(M)=\mathcal{V}_{H} \cap \mathcal{V}_{A}(M)$.

For infinitesimal group schemes of height 1 , one can make the descriptions of support varieties quite explicit. Let $H$ be an affine algebraic group scheme defined over $\mathbb{F}_{p}, H_{1}=\operatorname{ker}\left(\left.F\right|_{H}\right)$ and $\mathfrak{h}=$ Lie $H$ (which is a restricted Lie algebra with $[p$ ] operator). Let $\mathcal{N}_{1}(\mathfrak{h})$ be the closed subvariety of nilpotent elements in $\mathfrak{h}$ of $H$ defined by

$$
\mathcal{N}_{1}(\mathfrak{h}):=\left\{x \in \mathfrak{h}: x^{[p]}=0\right\} .
$$

We have the following identification of varieties.

Proposition 3.2 [16, (1.6), (5.11)]. $\mathcal{V}_{H_{1}}$ is homeomorphic to $\mathcal{N}_{1}(\mathfrak{h})$.

Finally, we can use the identification in Proposition 3.1 to identify $\mathcal{V}_{H_{1}}(M)$ as a closed subvariety of $\mathcal{N}_{1}(\mathfrak{h})$.

Proposition 3.3 [8, Theorem 1.3]. $\mathcal{V}_{H_{1}}(M)$ is homeomorphic to

$$
\left\{x \in \mathcal{N}_{1}(\mathfrak{h}): M \text { is not x-projective }\right\} \cup\{0\} .
$$

For the purposes of this paper, we need to analyze the relationship of support varieties over $B_{r}$ versus $P_{r}$, where $P$ is a parabolic subgroup of $G$. The following result is a generalization of [8, Theorem 1.2] and [2, Proposition 4.5.2].

Theorem 3.4. Let $J \subseteq \Delta$, let $P=P_{J}$ be the associated parabolic subgroup and let $M \in \bmod (P)$. Then

$$
\mathcal{V}_{P_{r}}(M)=P \cdot \mathcal{V}_{B_{r}}(M) .
$$

Proof. The proof follows along the same line of reasoning as in [8, Theorem (1.2)]. We will indicate what modifications are necessary. Let $\Psi=$ res $^{*}: \mathcal{V}_{B_{r}}(M) \rightarrow \mathcal{V}_{P_{r}}(M)$ be the map on varieties induced from the restriction map res : $\mathrm{H}^{\bullet}\left(P_{r}, k\right) \rightarrow \mathrm{H}^{\bullet}\left(B_{r}, k\right)$. According to $[16,(1.6),(5.11)]$, we can identify $\mathcal{V}_{B_{r}}(M)$ with $\Psi\left(\mathcal{V}_{B_{r}}(M)\right)$ in $\mathcal{V}_{P_{r}}(M)$. Since $\mathcal{V}_{P_{r}}(M)$ is invariant under $P$, we have

$$
P \cdot \mathcal{V}_{B_{r}}(M) \subseteq \mathcal{V}_{P_{r}}(M) .
$$

We need to show that the reverse inclusion holds.

Following the proof of [8, Theorem 1.2], set

$$
\begin{aligned}
I_{M} & =\operatorname{ker}\left\{\mathrm{H}^{\bullet}\left(B_{r}, k\right) \rightarrow \operatorname{Ext}_{B_{r}}^{\bullet}(M, M)\right\}, \\
J_{M} & =\operatorname{ker}\left\{\mathrm{H}^{\bullet}\left(P_{r}, k\right) \rightarrow \operatorname{Ext}_{P_{r}}^{\bullet}(M, M)\right\}, \\
K_{M} & =\left\{x \in \mathrm{H}^{\bullet}\left(P_{r}, k\right): p \cdot \operatorname{res}(x) \in I_{M}, \quad \forall p \in P\right\} \\
L_{M} & =\left\{x \in \mathrm{H}^{\bullet}\left(P_{r}, k\right): p \cdot \operatorname{res}(x) \in \sqrt{I_{M}} \forall p \in P\right\} .
\end{aligned}
$$


In the proof, if one replaces ' $G$ ' by ' $P$ ', removes the 'symmetric algebras' and uses the fact that $H^{m}(P / B,-)=0$ for $m>\operatorname{dim} P / B$, then one can conclude that $K_{M} \subseteq \sqrt{J_{M}}$. Thus, $\mathcal{V}_{P_{r}}(M) \subseteq P \cdot \mathcal{V}_{B_{r}}(M)$.

For $M$ a rational $B$-module, the relationship between the (relative) $B_{r}$ support variety of a module induced from $M$ and the $G_{r}$ support variety is described in [14, Theorem 5.4.1]. We generalize this result to the parabolic case as follows.

Theorem 3.5. Let $M$ be a rational B-module and $P$ be a parabolic subgroup of $G$ that contains $B$. Suppose that $t$ is a fixed integer and that $R^{m} \operatorname{ind}_{B}^{P} M=0$ for all $m \neq t$. Then

$$
\mathcal{V}_{P_{r}}\left(R^{t} \operatorname{ind}_{B}^{P} M\right)=P \cdot \mathcal{V}_{B_{r}}\left(R^{t} \operatorname{ind}_{B}^{P} M, M\right) .
$$

Proof. The proof of [14, Theorem 5.4.1] is formal and carries over after replacing $G$ by $P$. The main issue involves the use of a spectral sequence, which in our case is

$$
E_{2}^{m, n}=R^{m} \operatorname{ind}_{B / B_{r}}^{P / P_{r}} \operatorname{Ext}_{B_{r}}^{n}\left(R^{t} \operatorname{ind}_{B}^{P} M, M\right) \Rightarrow \operatorname{Ext}_{P_{r}}^{m+n-t}\left(R^{t} \operatorname{ind}_{B}^{P} M, R^{t} \operatorname{ind}_{B}^{P} M\right),
$$

and an increasing filtration whose finiteness depends on a vanishing result,

$$
R^{m} \operatorname{ind}_{B / B_{r}}^{P / P_{r}}=0 \quad \forall m>\operatorname{dim} P / B .
$$

This vanishing result holds by Proposition 2.2(ii).

\section{4. $G$-saturation}

We are interested in determining the support varieties $\mathcal{V}_{B_{1}}\left(H^{0}(X(w), \mathcal{L}(\lambda))\right)$ for all $w \in W$ and $\lambda \in X_{+}$. In particular, we want to understand the inclusion relations among support varieties for different $w$ and $\lambda$ of particular interest. In some instances we will use $H^{0}(w, \lambda):=H^{0}(X(w), \mathcal{L}(\lambda))$ as a shorthand notation. In the following theorem, we prove that for a fixed weight $\lambda$, the inclusion relation on the $G$-saturation of support varieties for Demazure modules respects the Bruhat order on $W$.

Theorem 4.1. Let $\lambda \in X_{+}$and $w_{1}<w_{2}$ in the Bruhat order on W. Then

$$
G \cdot \mathcal{V}_{B_{r}}\left(H^{0}\left(w_{2}, \lambda\right)\right) \subseteq G \cdot \mathcal{V}_{B_{r}}\left(H^{0}\left(w_{1}, \lambda\right)\right) .
$$

Proof. By induction on $\ell\left(w_{2}\right)-\ell\left(w_{1}\right)$, it suffices to prove the result when $w_{2}=s_{\alpha} w_{1}$ and $\ell\left(w_{2}\right)=\ell\left(w_{1}\right)+1$. Let $P_{\{\alpha\}}$ be the minimal parabolic subgroup corresponding to $\alpha$. By Theorem 3.4,

$$
\mathcal{V}_{\left(P_{\{\alpha\}}\right)_{r}}\left(H^{0}\left(w_{2}, \lambda\right)\right)=P_{\{\alpha\}} \cdot \mathcal{V}_{B_{r}}\left(H^{0}\left(w_{2}, \lambda\right)\right) .
$$

Since $H^{0}\left(w_{2}, \lambda\right) \cong \operatorname{ind}_{B}^{P_{\{\alpha\}}} H^{0}\left(w_{1}, \lambda\right)$, Theorem 3.5 implies

$$
\begin{aligned}
P_{\{\alpha\}} \cdot \mathcal{V}_{B_{r}}\left(H^{0}\left(w_{2}, \lambda\right)\right) & =P_{\{\alpha\}} \cdot \mathcal{V}_{B_{r}}\left(H^{0}\left(w_{2}, \lambda\right), H^{0}\left(w_{1}, \lambda\right)\right) \\
& \subseteq P_{\{\alpha\}} \cdot \mathcal{V}_{B_{r}}\left(H^{0}\left(w_{1}, \lambda\right)\right)
\end{aligned}
$$


TABle 1. Support varieties for Demazure modules in type $A_{2}$ with highest weight $(p-1) \rho$.

\begin{tabular}{cc}
\hline$w$ & $\mathcal{V}_{B_{1}}\left(H^{0}(w,(p-1) \rho)\right)$ \\
\hline$e$ & $\mathfrak{u}$ \\
$s_{\alpha}$ & $\mathfrak{u}_{\{\alpha\}}$ \\
$s_{\beta}$ & $\mathfrak{u}_{\{\beta\}}$ \\
$s_{\alpha} s_{\beta}$ & $\mathfrak{H}_{\{\alpha\}} \cup \mathfrak{u}_{\{\beta\}}$ \\
$s_{\beta} s_{\alpha}$ & $\mathfrak{H}_{\{\alpha\}} \cup \mathfrak{u}_{\{\beta\}}$ \\
$w_{0}$ & $\{0\}$ \\
\hline
\end{tabular}

Combining (4.1) and (4.2), we have

$$
\mathcal{V}_{\left(P_{\{\alpha\}}\right)_{r}}\left(H^{0}\left(w_{2}, \lambda\right)\right) \subseteq P_{\{\alpha\}} \cdot \mathcal{V}_{B_{r}}\left(H^{0}\left(w_{1}, \lambda\right)\right),
$$

so acting by $G$ on both sides we certainly have

$$
G \cdot \mathcal{V}_{\left(P_{\{\alpha\}}\right)_{r}}\left(H^{0}\left(w_{2}, \lambda\right)\right) \subseteq G \cdot \mathcal{V}_{B_{r}}\left(H^{0}\left(w_{1}, \lambda\right)\right) .
$$

Finally, by Proposition 3.1, $\mathcal{V}_{B_{r}}(M) \subseteq \mathcal{V}_{\left(P_{\{\alpha\}}\right)_{r}}(M)$ for all $M \in \bmod \left(P_{\{\alpha\}}\right)$. Thus,

$$
G \cdot \mathcal{V}_{B_{r}}\left(H^{0}\left(w_{2}, \lambda\right)\right) \subseteq G \cdot \mathcal{V}_{B_{r}}\left(H^{0}\left(w_{1}, \lambda\right)\right),
$$

as required.

We should remark that the result above is rather subtle in the sense that inclusion of the $B_{1}$-support varieties of Demazure modules need not be preserved under the Bruhat order. This can be seen in the following example.

ExAmple 4.2. Let $p \geq 3, \lambda=(p-1) \rho$ (the Steinberg weight) and $G=\operatorname{SL}(3)$. Let $\mathfrak{u}_{\{\alpha\}}$ and $\mathfrak{u}_{\{\beta\}}$ be the unipotent radical of the Lie algebras of $P_{\{\alpha\}}$ and $P_{\{\beta\}}$. The computation in Section 6 gives the support varieties $\mathcal{V}_{B_{1}}\left(H^{0}(w,(p-1) \rho)\right)$ for all $w \in W$, see Table 1 .

In particular, the pair $s_{\beta}$ and $s_{\alpha} s_{\beta}$ illustrates that $w_{1}<w_{2}$ does not necessarily imply that $\mathcal{V}_{B_{1}}\left(H^{0}\left(w_{2}, \lambda\right)\right) \subseteq \mathcal{V}_{B_{1}}\left(H^{0}\left(w_{1}, \lambda\right)\right)$. Note, however, that the saturations in these two cases agree:

$$
G \cdot \mathfrak{u}_{\{\beta\}}=G \cdot \mathfrak{u}_{\{\alpha\}}=G \cdot\left(\mathfrak{u}_{\{\alpha\}} \cup \mathfrak{u}_{\{\beta\}}\right) .
$$

Fix a dominant weight $\lambda$. The subset

$$
\Phi_{\lambda, p}=\left\{\alpha \in \Phi^{+}:\left(\lambda+\rho, \alpha^{\vee}\right) \in p \mathbb{Z}\right\}
$$

is a subroot system of $\Phi$, which, when the prime $p$ is good relative to $\Phi$, is conjugate under the Weyl group to a root system $\Phi_{I}$ spanned by a subset $I \subseteq \Delta$ of simple roots, see [5, Proposition 24, p. 165]. The weight $\lambda$ is called p-regular if $\Phi_{\lambda, p}=\emptyset$.

Proposition 4.3. Let $\lambda$ be a p-regular weight in $X_{+}$. Then $\mathcal{V}_{B_{1}}\left(H^{0}(w, \lambda)\right)=\mathcal{V}_{B_{1}}$. 
Proof. If $w_{0}$ denotes the longest element of the Weyl group, then $w \leq w_{0}$, so Theorem 4.1 gives us an inclusion of the saturated supports:

$$
G \cdot \mathcal{V}_{B_{1}}\left(H^{0}\left(w_{0}, \lambda\right)\right) \subseteq G \cdot \mathcal{V}_{B_{1}}\left(H^{0}(w, \lambda)\right) .
$$

Since $X\left(w_{0}\right)=G / B, H^{0}\left(w_{0}, \lambda\right)$ is a $G$-module and

$$
\mathcal{V}_{G_{1}}\left(H^{0}\left(w_{0}, \lambda\right)\right)=\mathcal{V}_{G_{1}}\left(H^{0}(G / B, \mathcal{L}(\lambda))\right) .
$$

Moreover, by [8, Theorem 1.2], $\mathcal{V}_{G_{1}}\left(H^{0}(G / B, \mathcal{L}(\lambda))\right)=G \cdot \mathcal{V}_{B_{1}}\left(H^{0}(G / B, \mathcal{L}(\lambda))\right)$. Putting these results together, we have

$$
\mathcal{V}_{G_{1}}\left(H^{0}\left(w_{0}, \lambda\right)\right) \subseteq G \cdot \mathcal{V}_{B_{1}}\left(H^{0}(w, \lambda)\right) .
$$

Since $\lambda$ is $p$-regular, $\mathcal{V}_{G_{1}}\left(H^{0}\left(w_{0}, \lambda\right)\right)=\mathcal{V}_{G_{1}}$ by [14, Proposition 4.1.2] and thus

$$
\mathcal{V}_{G_{1}} \subseteq G \cdot \mathcal{V}_{B_{1}}\left(H^{0}(w, \lambda)\right) \subseteq \mathcal{V}_{G_{1}} .
$$

Therefore, we must have

$$
G \cdot \mathcal{V}_{B_{1}}\left(H^{0}(w, \lambda)\right)=\mathcal{V}_{G_{1}} .
$$

Since $\lambda$ is $p$-regular, we have $p \geq h$ (see [12, Section II 6.2(10)]), and $\mathcal{V}_{G_{1}}$ identifies with the nilpotent cone in $\mathfrak{g}$. Therefore, the closed conical $B$-stable variety $\mathcal{V}_{B_{1}}\left(H^{0}(w, \lambda)\right)$ must contain a regular nilpotent element. It follows that

$$
\mathcal{V}_{B_{1}}\left(H^{0}(w, \lambda)\right)=\mathfrak{u}=\mathcal{N}_{1}(\mathfrak{u})=\mathcal{V}_{B_{1}},
$$

as required.

We conclude this section by illustrating Proposition 4.3 in the situation when the root system $\Phi$ is $A_{1}$ (that is, for the group $G=\operatorname{SL}(2)$ ).

Let $G=\operatorname{SL}(2)$ and $\lambda$ be a dominant integral weight (represented by a nonnegative integer). In this case, $G / B \cong \mathbb{P}^{1}$ and $W=\left\{e, s_{\alpha}\right\}$.

Let $w=e$. We have $X(e)=e B / B$, which consists of a single point. It follows that $\operatorname{dim} H^{0}(w, \lambda)=1$ and so by the rank variety description, $\mathcal{V}_{B_{1}}\left(H^{0}(w, \lambda)\right)=\mathfrak{u}$, which is independent of $\lambda$.

If $w=s_{\alpha}$ is the long element of $W$, then $X\left(s_{\alpha}\right)=G / B$. Now the weight $\lambda$ is $p$ regular if and only if $p \nmid \lambda+1$. So, by Proposition 4.3, $\mathcal{V}_{B_{1}}\left(H^{0}\left(s_{\alpha}, \lambda\right)\right)=\mathcal{V}_{B_{1}}=\mathfrak{u}$ unless $p \mid \lambda+1$. When $p \mid \lambda+1$, a simple application of [14, Theorem 6.2.1] gives

$$
\mathcal{V}_{B_{1}}\left(H^{0}(w, \lambda)\right)=\{0\} .
$$

We summarize the situation for type $A_{1}$ in Table 2 .

\section{Calculation of support varieties}

In this section, we determine the support varieties of Demazure modules for arbitrary reductive groups $G$ when the underlying Schubert scheme corresponds either to the longest element in $W_{I}$ (for any $I \subseteq \Delta$ ) or to the longest element in $W^{J}$ (for certain subsets $J \subseteq \Delta$ ). 
TABLE 2. Calculation of support varieties for all Demazure modules in type $A_{1}$.

\begin{tabular}{ccc}
\hline$w$ & $p \nmid \lambda+1$ & $p \mid \lambda+1$ \\
\hline$e$ & $\mathfrak{u}$ & $\mathfrak{u}$ \\
$s_{\alpha}$ & $\mathfrak{u}$ & $\{0\}$ \\
\hline
\end{tabular}

Let $\lambda \in X_{+}$and define

$$
J_{\lambda}=\left\{\alpha \in \Delta:\left\langle\lambda, \alpha^{\vee}\right\rangle=0\right\} .
$$

For any subset $J \subseteq \Delta$, let $w_{0, J}$ denote the Weyl group element of maximal length in $W^{J}$.

Proposition 5.1. Let $\lambda \in X_{+}$, let $P=P_{J_{\lambda}}$ and let $w=w_{0, J_{\lambda}}$. Then there is an isomorphism of $B$-modules

$$
H^{0}\left(X(w)_{P}, \mathcal{L}(\lambda)\right) \cong H^{0}(G / B, \mathcal{L}(\lambda))
$$

Proof. The resolution diagram (2.1) induces a diagram involving cohomology groups.

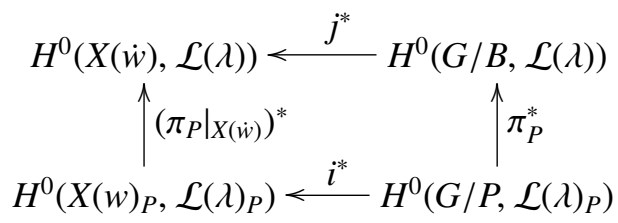

By Proposition 2.1, $\left(\left.\pi_{P}\right|_{X(\dot{w})}\right)^{*}$ is an isomorphism. Also, the choice of $w$ implies that $X(w)_{P}=G / P$, hence $i$ is the identity and $i^{*}$ is an isomorphism. By local triviality (see [12, Section I 5.17]), the map $\pi_{P}^{*}$ is an isomorphism. The diagram commutes and so $j^{*}$ is an isomorphism.

The proposition and [14, Theorem 6.2.1] allow us to identify the support variety of $H^{0}\left(X(w)_{P}, \mathcal{L}(\lambda)_{P}\right)$ in this special case. Choose $x \in W$ such that $x\left(\Phi_{\lambda, p}\right)=\Phi_{I}$ for some subset $I \subseteq \Delta$.

Theorem 5.2. With $J=J_{\lambda}, w=w_{0, J}$ and $P=P_{J}$ as above,

$$
\mathcal{V}_{B_{1}}\left(H^{0}\left(X(w)_{P}, \mathcal{L}(\lambda)_{P}\right)\right)=\left(G \cdot \mathfrak{u}_{I}\right) \cap \mathcal{N}_{1}(\mathfrak{u}) .
$$

Proof. By [14, Theorem 6.2.1], $\mathcal{V}_{G_{1}}\left(H^{0}(G / B, \mathcal{L}(\lambda))\right)=G \cdot \mathfrak{u}_{I}$. The isomorphism of Proposition 5.1 along with naturality of supports, see (3.1.1), implies that

$$
\begin{aligned}
\mathcal{V}_{B_{1}}\left(H^{0}\left(X(w)_{P}, \mathcal{L}(\lambda)_{P}\right)\right) & =\mathcal{V}_{B_{1}}\left(H^{0}(G / B, \mathcal{L}(\lambda))\right) \\
& =\mathcal{V}_{G_{1}}\left(H^{0}(G / B, \mathcal{L}(\lambda))\right) \cap \mathcal{N}_{1}(\mathfrak{u}) \\
& =\left(G \cdot \mathfrak{u}_{I}\right) \cap \mathcal{N}_{1}(\mathfrak{u}),
\end{aligned}
$$

as claimed. 
Theorem 5.2 implies that the $B_{1}$ support varieties of certain Demazure modules are unions of the closures of orbital varieties. Recall from the introduction that the $B_{1}$ support varieties of induced modules $H^{0}(G / B, \mathcal{L}(\lambda))$ are also unions of orbital variety closures. It remains an interesting open problem whether or not the support varieties of all Demazure modules are unions of orbital variety closures and whether one can realize all such closures as support varieties of certain modules.

Let $I \subseteq \Delta$ be an arbitrary subset and let $w=w_{I} \in W_{I}$ be such that $w_{I}(\alpha)<0$ for all $\alpha \in I$. The element $w_{I}$ is the long element in the group $W_{I}$. First, note that in this case by [12, Section II 13.3 (4)]

$$
H^{0}(X(w), \mathcal{L}(\lambda)) \cong \operatorname{ind}_{B}^{P_{I}} \lambda .
$$

Consequently, $H^{0}(X(w), \mathcal{L}(\lambda))$ is a $P_{I}$-module with $U_{I}$ acting trivially. The following theorem describes the support variety of $H^{0}(X(w), \mathcal{L}(\lambda))$ as a $\left(P_{I}\right)_{1}$-module by reducing to the case of [14, Theorem 6.2.1] for the Levi subgroup $L_{I}$.

Theorem 5.3. Let $I \subseteq \Delta$ with $\mathfrak{u}_{I}=$ Lie $U_{I}$, and $w=w_{I}$. Then

$$
\mathcal{V}_{\left(P_{I}\right)_{1}}\left(H^{0}(X(w), \mathcal{L}(\lambda))\right)=\left[\mathcal{V}_{\left(L_{I}\right)_{1}}\left(H_{I}^{0}(\lambda)\right)+\mathfrak{u}_{I}\right] \cap \mathcal{N}_{1}\left(\mathfrak{p}_{I}\right) .
$$

Proof. Set $\mathfrak{l}_{I}=$ Lie $L_{I}$ and $\mathfrak{u}_{I}=$ Lie $U_{I}$. First observe by [7, Examples 4.2] that

$$
\left.\operatorname{ind}_{B}^{P_{I}} \lambda\right|_{L_{I}} \cong \operatorname{ind}_{L_{I} \cap B}^{L_{I}} \lambda:=H_{I}^{0}(\lambda) \text {. }
$$

Let $z=x+y$, where $x \in \mathfrak{l}_{I}, y \in \mathfrak{u}_{I}$ and $z \in \mathcal{N}_{1}\left(\mathfrak{p}_{I}\right)$. Then, by [6, Proposition 5.2(a)], $x \in \mathcal{N}_{1}\left(\mathfrak{l}_{I}\right)$. Since $\mathfrak{u}_{I}$ acts trivially on $H_{I}^{0}(\lambda)$,

$$
z \cdot H_{I}^{0}(\lambda)=x \cdot H_{I}^{0}(\lambda)
$$

In particular, $H_{I}^{0}(\lambda)$ is $z$-projective if and only if it is $x$-projective. By the realization of the support varieties in terms of rank varieties, we can conclude that $z \in \mathcal{V}_{\left(P_{I}\right)_{1}}\left(H^{0}(X(w), \mathcal{L}(\lambda))\right)$ if and only if $x \in \mathcal{V}_{\left(L_{I}\right)_{1}}\left(H^{0}(X(w), \mathcal{L}(\lambda))\right)$.

Therefore,

$$
\mathcal{V}_{\left(P_{I}\right)_{1}}\left(H^{0}(X(w), \mathcal{L}(\lambda))\right)=\left[\mathcal{V}_{\left(L_{I}\right)_{1}}\left(H_{I}^{0}(\lambda)\right)+\mathfrak{u}_{I}\right] \cap \mathcal{N}_{1}\left(\mathfrak{p}_{I}\right),
$$

as desired.

Using [14, Theorem 6.2.1], we obtain the following description of the support variety. Recall that when the prime $p$ is good there exists $x \in W$ such that $x\left(\Phi_{\lambda, p}\right)=\Phi_{J}$ for some subset $J \subseteq \Delta$.

Corollary 5.4. Let $w=w_{I}$ as above, let $\lambda \in X(T)_{+}$and suppose $p$ is a good prime for $\Phi$. Let $x \in W_{I}$ be such that $x\left(\left(\Phi_{I}\right)_{\lambda, p}\right)=\left(\Phi_{I}\right)_{J}$ for some subset $J \subset I$. Let $\mathfrak{u}_{I, J}$ be the nilradical of the parabolic in $\mathfrak{l}_{I}$ corresponding to $J$. Then

$$
\begin{aligned}
\mathcal{V}_{\left(P_{I}\right)_{1}}\left(H^{0}(X(w), \mathcal{L}(\lambda))\right) & =\left(L_{I} \cdot \mathfrak{u}_{I, J}+\mathfrak{u}_{I}\right) \cap \mathcal{N}_{1}\left(\mathfrak{p}_{I}\right) \\
& =\left(L_{I} \cdot\left(\mathfrak{u}_{I, J}+\mathfrak{u}_{I}\right)\right) \cap \mathcal{N}_{1}\left(\mathfrak{p}_{I}\right) \\
& =\left(L_{I} \cdot \mathfrak{u}_{J}\right) \cap \mathcal{N}_{1}\left(\mathfrak{p}_{I}\right) .
\end{aligned}
$$


The explicit calculation of Corollary 5.4 and the inclusions among saturated support varieties in Theorem 4.1 give upper and lower bounds on the saturation $G \cdot \mathcal{V}_{B_{1}}\left(H^{0}(w, \lambda)\right)$ for arbitrary $w \in W$ and $\lambda \in X(T)_{+}$. To state the bounds obtained, we introduce some notation. For $v \in W$, let $v=s_{\gamma_{1}} \cdots s_{\gamma_{n}}$ be a reduced expression. Define the support of $v$ by $S(v)=\left\{\gamma_{1}, \ldots, \gamma_{n}\right\}$. This definition is independent of the reduced expression chosen (see [4, Theorem 3.3.1]). As in the previous section, $w_{I}$ denotes the long element of $W_{I}$ for a subset $I \subseteq \Delta$.

Lemma 5.5. If $v \in W$, then $v \leq w_{S(v)}$. Moreover, $v \leq w_{I}$ implies that $S(v) \subseteq I$ and $w_{S(v)} \leq w_{I}$.

Proof. This is a consequence of [11, Theorem 5.10]. The expression $v=s_{\gamma_{1}} \cdots s_{\gamma_{n}}$ implies that $v \in W_{S(v)}$ and hence $v \leq w_{S(v)}$, since the latter is the unique longest element of $W_{S(v)}$. Similarly, $v \leq w_{I}$ implies that the generators of $W_{S(v)}$ are contained in $W_{I}$ and hence $w_{S(v)} \leq w_{I}$.

The lemma gives us a precise characterization of the least upper bound by elements of the form $w_{I}$, where $I \subseteq \Delta$. In general, there is no unique greatest lower bound, as Example 5.6 shows.

ExAmple 5.6. Let $W$ be the Weyl group of type $A_{3}$ generated by simple reflections $s_{\alpha_{1}}, s_{\alpha_{2}}, s_{\alpha_{3}}$ such that $s_{\alpha_{1}}$ and $s_{\alpha_{3}}$ commute.

- The element $w=s_{\alpha_{1}} s_{\alpha_{2}}$ has support $S\left(s_{\alpha_{1}} s_{\alpha_{2}}\right)=\left\{s_{\alpha_{1}}, s_{\alpha_{2}}\right\}$ and its unique parabolic upper bound in the Bruhat order is $w_{\left\{\alpha_{1}, \alpha_{2}\right\}}=s_{\alpha_{1}} s_{\alpha_{2}} s_{\alpha_{1}}$. On the other hand, $w$ has maximal lower bounds $w_{\left\{\alpha_{1}\right\}}=s_{\alpha_{1}}$ and $w_{\left\{\alpha_{2}\right\}}=s_{\alpha_{2}}$ which are incomparable.

- $\quad$ The element $w=s_{\alpha_{1}} s_{\alpha_{2}} s_{\alpha_{3}}$ has support $S\left(s_{\alpha_{1}} s_{\alpha_{2}} s_{\alpha_{3}}\right)=\Delta$, so its unique upper bound is $w_{0}=s_{\alpha_{1}} s_{\alpha_{2}} s_{\alpha_{3}} s_{\alpha_{1}} s_{\alpha_{2}} s_{\alpha_{3}}$. The set of all parabolic elements bounded above by $w$ is $\left\{e, s_{\alpha_{1}}, s_{\alpha_{2}}, s_{\alpha_{3}}, s_{\alpha_{1}} s_{\alpha_{3}}\right\}$.

As an application, the explicit description of supports given by Corollary 5.4 implies the following explicit upper and lower bounds on the $G$-saturated support variety of a Demazure module.

Proposition 5.7. Let $v \in W$ and $\lambda \in X(T)_{+}$. Then

$$
G \cdot \mathcal{V}_{B_{1}}\left(H^{0}\left(w_{S(v)}, \lambda\right)\right) \subseteq G \cdot \mathcal{V}_{B_{1}}\left(H^{0}(v, \lambda)\right) \subseteq \bigcap_{w_{I} \leq v} G \cdot \mathcal{V}_{B_{1}}\left(H^{0}\left(w_{I}, \lambda\right)\right),
$$

where the intersection may be taken over the set of $w_{I} \leq v$ which are maximal with respect to that property.

Recall that the varieties of the form $\mathcal{V}_{B_{1}}\left(H^{0}\left(w_{I}, \lambda\right)\right)$ are explicitly determined in Corollary 5.4 . 
TABLE 3. $B_{1}$-support varieties for $A_{2}$ when $\ell(w) \neq 2, p \geq 3$.

\begin{tabular}{ccl}
\hline$w$ & $\mathcal{V}_{B_{1}}\left(H^{0}(w, \lambda)\right)$ & $\lambda$ \\
\hline$e$ & $\mathfrak{u}$ & All $\lambda$ \\
$s_{\alpha}$ & $\mathfrak{u}_{\{\alpha\}}$ & $p \mid \lambda_{1}+1$ \\
& $\mathfrak{u}$ & $p \nmid \lambda_{1}+1$ \\
$s_{\beta}$ & $\mathfrak{u}_{\{\beta\}}$ & $p \mid \lambda_{2}+1$ \\
& $\mathfrak{u}$ & $p \nmid \lambda_{2}+1$ \\
$s_{\alpha} s_{\beta} s_{\alpha}$ & $\mathcal{V}_{G_{1}}\left(H^{0}(\lambda)\right) \cap \mathfrak{u}$ & All $\lambda$ \\
\hline
\end{tabular}

\section{Support varieties of Demazure modules for the root system $A_{2}$}

In this section, we present explicit calculations of the support varieties for Demazure modules when the group $G$ has a root system of type $A_{2}$. We proceed by applying our results from Section 5 in the case when the prime $p$ is good. For type $A_{2}$, this means that $p \geq 3$. We return to the case when $p=2$ at the end of the section.

Let $G=\operatorname{SL}(3)$ with $p \geq 3$, and let $\lambda=\left(\lambda_{1}, \lambda_{2}\right)$ be a dominant integral weight expressed in terms of the fundamental weights. Let us identify $\Delta=\{\alpha, \beta\}$ and $W=$ $\left\{e, s_{\alpha}, s_{\beta}, s_{\alpha} s_{\beta}, s_{\beta} s_{\alpha}, s_{\alpha} s_{\beta} s_{\alpha}\right\}$. The cases where $\ell(w) \neq 2$, that is, $w \in\left\{e, s_{\alpha}, s_{\beta}, s_{\alpha} s_{\beta} s_{\alpha}\right\}$, are covered by Corollary 5.4. For such a $w$, set $\mathcal{V}=\mathcal{V}_{B_{1}}\left(H^{0}(w, \lambda)\right)$. We summarize this in Table 3.

In the $w=s_{\alpha} s_{\beta} s_{\alpha}$ case, $J \subset \Delta$ depends on $\lambda$ and $p$ as in the discussion before Corollary 5.4.

For the cases where $\ell(w)=2$, we analyze the regularity of $\lambda$ with respect to the prime $p$ and $p$-divisibility of the dimension of $H^{0}(w, \lambda)$. We treat the case $w=s_{\alpha} s_{\beta}$, the other case being symmetric upon switching $\alpha, \beta$ and $\lambda_{1}, \lambda_{2}$. For convenience, denote by $M(\lambda)=M\left(\lambda_{1}, \lambda_{2}\right)$ the $B$-module $H^{0}\left(s_{\alpha} s_{\beta}, \lambda\right)$, which we also identify with $\operatorname{ind}_{B}^{P_{\{\alpha\}}} \operatorname{ind}_{B}^{P_{\{\beta\}}} \lambda$.

In the root system of type $A_{2}$, a weight $\lambda$ is $p$-regular if and only if

$$
\left\{\begin{array}{l}
p \nmid \lambda_{1}+1, \\
p \nmid \lambda_{2}+1, \\
p \nmid \lambda_{1}+\lambda_{2}+2 .
\end{array}\right.
$$

We may apply the Demazure character formula (see [1]) in this situation to conclude that $\operatorname{dim} M(\lambda)=\left(\lambda_{2}+1\right)\left(2 \lambda_{1}+\lambda_{2}+2\right) / 2$. Thus, $p$ does not divide $\operatorname{dim} M(\lambda)$ if and only if

$$
\left\{\begin{array}{l}
p \nmid \lambda_{2}+1, \\
p \nmid 2 \lambda_{1}+\lambda_{2}+2 .
\end{array}\right.
$$


THEOREM 6.1. Let $p \geq 3$. The $B_{1}$-support variety $\mathcal{V}=\mathcal{V}_{B_{1}}(M(\lambda))$ is $\mathfrak{u}$ if either (6.1) or (6.2) hold. Otherwise, $\mathcal{V}$ is a proper subvariety of $\mathfrak{u}$ given by the conditions below:

$$
\mathcal{V}_{B_{1}}(M(\lambda))= \begin{cases}\mathfrak{u}_{\{\alpha\}} & \text { if } \lambda=(n p-1,0), \text { where } n \geq 1, \\ \mathfrak{u}_{\{\alpha\}} \cup \mathfrak{u}_{\{\beta\}} & \text { if } \lambda_{2} \neq 0 \text { and neither (6.1) nor (6.2) hold }\end{cases}
$$

The rest of the section is devoted to proving Theorem 6.1. First, if either (6.1) or (6.2) holds, then we conclude that $\mathcal{V}=\mathfrak{u}$ by Proposition 4.3 or the rank variety description of $\mathcal{V}$, respectively. For the rest of the section, we assume that neither (6.1) nor (6.2) holds and calculate $\mathcal{V}$, which will turn out to be a proper subvariety of $\mathfrak{u}$.

Our analysis uses the $B$-stability of support varieties in a crucial way, in particular the action of positive root subgroups and certain one-parameter groups in the maximal torus. The nilradical $\mathfrak{u}$ is spanned by root spaces $\mathfrak{u}=k X_{\alpha} \oplus k X_{\beta} \oplus k X_{\alpha+\beta}$. There is a one-parameter subgroup $k^{*} \subset T \subset B$ such that

$$
t \cdot X_{\gamma}=t^{\mathrm{ht}(\gamma)} X_{\gamma}
$$

for all $t \in k^{*}$ and $\gamma \in \Phi$. This group is generated by the element usually denoted by $H_{\rho}$, where $\rho$ is the half sum of positive roots $(\rho=\alpha+\beta$ in this case).

As a preliminary step, we classify the $\left(B, k^{*}\right)$-stable subvarieties of $\mathfrak{u}$. Let $v=$ $a X_{\alpha}+b X_{\beta}+c X_{\alpha+\beta}$ be an arbitrary point of $X \subset \mathfrak{u}$, an irreducible $B$-stable subvariety of $\mathfrak{u}$. Here, rank $v$ denotes the rank of a matrix representative for $v$. The claim is that $X$ is equal to one of the following ( $B$-stable) subspaces: $\mathfrak{u}, \overline{B \cdot X_{\alpha}}=k X_{\alpha} \oplus k X_{\alpha+\beta}$, $\overline{B \cdot X_{\beta}}=k X_{\beta} \oplus k X_{\alpha+\beta}, \overline{B \cdot X_{\alpha+\beta}}=k X_{\alpha+\beta}$ or $\{0\}$. There are five mutually exclusive cases. (1) Suppose that $a, b \neq 0$. Then rank $v=2$ and the $B$-orbit through $v$ is dense in $\mathfrak{u}$. Thus, $X=\mathfrak{u}$.

(2) Suppose that $a \neq 0, b=0$. Using the action of $k^{*}$, we see that the element $v^{\prime}=a X_{\alpha}$ is in the closure of $B \cdot v$. Hence, $X_{\alpha} \in X$ and so $\overline{B \cdot X_{\alpha}} \subset X$.

(3) Suppose that $a=0, b \neq 0$. Then, as in the previous case, we conclude that $\overline{B \cdot X_{\beta}} \subset X$.

(4) Suppose that $a, b=0$ and $c \neq 0$. In this case, $\overline{B \cdot X_{\alpha+\beta}} \subset X$.

(5) Suppose that $a, b, c=0$. Then $v=0$ and $\{0\} \subset X$.

Therefore, every $B$-stable, irreducible subvariety $X \subset \mathfrak{u}$ is a union of the five subspaces above; thus, it must equal one of them.

Now we treat a number of cases depending on $\lambda$ and $p$ to determine which root vectors are in the support variety. By the analysis of the previous paragraph, this suffices to determine the variety as a union of $B$-stable subvarieties.

First, suppose $\lambda_{2}=0$. In this case, $\operatorname{ind}_{B}^{P_{(\beta)}} \lambda \cong \lambda$ as a $B$-module and so $M\left(\lambda_{1}, 0\right) \cong$ $\operatorname{ind}_{B}^{P_{|\alpha|}}\left(\lambda_{1}, 0\right)$. Thus, by the $\ell(w)=1$ calculation in Table 3 ,

$$
\mathcal{V}_{B_{1}}\left(M\left(\lambda_{1}, 0\right)\right)= \begin{cases}\mathfrak{u}_{\{\alpha\}} & \text { if } p \mid \lambda_{1}+1, \\ \mathfrak{u} & \text { if } p \nmid \lambda_{1}+1 .\end{cases}
$$


This proves the first part of (6.3). Note that if $p$ and $\lambda$ are such that $p \nmid \lambda_{1}+1$ and $\lambda_{2}=0$, then they satisfy both (6.1) and (6.2).

Now suppose $\lambda_{2} \neq 0 . M(\lambda)$ is induced from $H^{0}\left(s_{\beta}, \lambda\right)$ as a $P_{\{\alpha\}}$-module and as an $L_{\{\alpha\}}$-module we have

$$
\left.M(\lambda)\right|_{L_{\{\alpha\}}} \cong \bigoplus_{i=1}^{\lambda_{2}+1} \operatorname{ind}_{B \cap L_{\{\alpha\}}}^{L_{\{\alpha\}}}\left(\lambda_{1}+i\right),
$$

where the right-hand side is a direct sum of irreducible $L_{\{\alpha\}}$-modules indexed by the integers $\lambda_{1}+1, \ldots, \lambda_{1}+\lambda_{2}+2$. The assumption that $\lambda_{2} \neq 0$ implies that $M(\lambda)$ has at least two $L_{\{\alpha\}}$ summands whose dimensions differ by 1 and so it cannot be projective over $\left\langle X_{\alpha}\right\rangle$. Therefore, $X_{\alpha} \in \mathcal{V}$ and by the analysis above of the $B$-stable, conical subvarieties of $\mathfrak{u}$, we get $\overline{B \cdot X_{\alpha}} \subset \mathcal{V}$. Thus, $X_{\alpha+\beta} \in \overline{B \cdot X_{\alpha}} \subset \mathcal{V}$. Using the fact that $\mathcal{V}$ is $P_{\{\alpha\}}$-stable, we can conclude that $X_{\beta} \in \overline{P_{\{\alpha\}} \cdot X_{\alpha+\beta}} \subset \mathcal{V}$. Hence, independent of $p$, we have

$$
\overline{B \cdot X_{\alpha}} \cup \overline{B \cdot X_{\beta}}=\mathfrak{u}_{\{\alpha\}} \cup \mathfrak{u}_{\{\beta\}} \subseteq \mathcal{V} .
$$

Suppose that $p \mid\left(\lambda_{2}+1\right)$. In this case, $\lambda_{2}$ is a Steinberg weight for $L_{\{\beta\}}$ and we have $\mathcal{V}_{B_{1}}\left(H^{0}\left(s_{\beta}, \lambda\right)\right)=\mathfrak{u}_{\{\beta\}}$. By Theorem 3.4,

$$
\mathcal{V}_{\left(P_{\{\alpha\}}\right)_{1}}(M(\lambda)) \subseteq P_{\{\alpha\}} \cdot \mathcal{V}_{B_{1}}\left(H^{0}\left(s_{\beta}, \lambda\right)\right)=P_{\{\alpha\}} \cdot \mathfrak{u}_{\{\beta\}} \cdot
$$

Now, observe that the right-hand side is contained in the subvariety

$$
R_{1}:=\{v \in \mathfrak{g}: \operatorname{rank} v \leq 1\} .
$$

Since $\operatorname{rank}\left(X_{\alpha}+X_{\beta}\right)=2$, we have that $\left(X_{\alpha}+X_{\beta}\right) \notin \mathcal{V}$, so $\mathcal{V}$ is a proper subvariety of $\mathfrak{u}$. We conclude in this case that

$$
\mathcal{V}=\mathfrak{u}_{\{\alpha\}} \cup \mathfrak{u}_{\{\beta\}} .
$$

We continue the proof of Theorem 6.1 with all the prior assumptions; in particular, $w=s_{\alpha} s_{\beta}$. Now assuming that $\lambda_{2} \neq 0$ and $p \nmid \lambda_{2}+1$, we reduce to two families of modules which also satisfy neither (6.1) nor (6.2).

Lemma 6.2. If $\lambda=\left(\lambda_{1}, \lambda_{2}\right)$ satisfy $\lambda_{2} \neq 0, p \nmid \lambda_{2}+1$, and neither (6.1) nor (6.2), then either

$$
\lambda_{1} \equiv-1 \bmod p, \quad \lambda_{2} \equiv 0 \bmod p
$$

or

$$
\lambda_{1} \equiv 0 \bmod p, \quad \lambda_{2} \equiv-2 \bmod p .
$$

Proof. First, if $\lambda$ and $p$ violate condition (6.2) and $p \nmid \lambda_{2}+1$, then $p \mid 2 \lambda_{1}+\lambda_{2}+2$. Since the pair also violates (6.1), there are two possibilities.

First, suppose that $p \mid \lambda_{1}+1$. Then $p \mid\left(2 \lambda_{1}+\lambda_{2}+2\right)=2\left(\lambda_{1}+1\right)+\lambda_{2}$ if and only if $p \mid \lambda_{2}$. This is case (i) above.

Otherwise, suppose that $p \mid \lambda_{1}+\lambda_{2}+2$. Then $p \mid\left(2 \lambda_{1}+\lambda_{2}+2\right)=\left(\lambda_{1}+\lambda_{2}+2\right)+\lambda_{1}$ if and only if $p \mid \lambda_{1}$. Hence, $p \mid \lambda_{2}+2$. These two conditions are equivalent to case (ii) above. 
To complete the proof, we make two reductions. First, we prove that if the support varieties for all modules of type (i) in Lemma 6.2 are proper, then the support varieties for all modules of type (ii) are proper, and vice versa. Next, we show by induction that it suffices to prove the properness of the support varieties for modules of the form $M(n p, p-2)$ for $n \geq 0$. These are modules of type (ii). Finally, we analyze the support of $M(n p, p-2)$ using filtrations on the tensor product $M(n p, m p-2) \otimes L(0,1)^{(1)}$, where $L(0,1)^{(1)}$ denotes the $G$-module $L(0,1)$ (with highest weight $\mu=(0,1)$ ) twisted once by the Frobenius morphism.

Lemma 6.3. The support $\mathcal{V}_{B_{1}}(M(\lambda)$ ) is a proper subvariety of $\mathfrak{u}$ (and hence equal to $\mathfrak{u}_{\{\alpha\}} \cup \mathfrak{u}_{\{\beta\}}$ ) for all $\lambda$ of type (i) if and only if the same holds for all $\lambda$ of type (ii).

Proof. Consider the $B$-module $M(n p, m p-1)$ for some $n \geq 0, m>0$. This module has proper support by the argument given for the case $p \mid \lambda_{2}+1$. Let $L(1,0)$ denote the irreducible $G$-module with highest weight $(1,0)$ and consider the tensor product $M(n p, m p-1) \otimes L(1,0)$. The $G$-module structure on $L(1,0)$ allows one to use the tensor identity [12, Section I 4.8] to identify

$$
\begin{aligned}
& M(n p, m p-1) \otimes L(1,0)=\left[\operatorname{ind}_{B}^{P_{\{\alpha\}}} \operatorname{ind}_{B}^{P_{\{\beta\}}}(n p, m p-1)\right] \otimes L(1,0) \\
& \cong \operatorname{ind}_{B}^{P_{\langle\alpha\rangle}} \operatorname{ind}_{B}^{P_{\{\beta\}}}[(n p, m p-1) \otimes L(1,0)] \text {. }
\end{aligned}
$$

Now, $L(1,0)$ has a filtration as a $B$-module as follows:

$$
L(1,0)=\left[\begin{array}{l}
(1,0) \\
(-1,1) \\
(0,-1)
\end{array} \text {, which induces }(n p, m p-1) \otimes L(1,0)=\left.\right|_{(n p-1, m p)} ^{(n p+1, m p-1)}\right.
$$

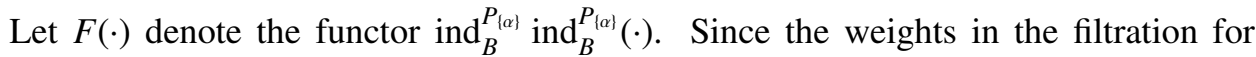
$(n p, m p-1) \otimes L(1,0)$ are all dominant, Kempf's vanishing theorem implies that $R^{1} F(\cdot)$ vanishes on each of the subquotients (see [12, Section I 4.4]). Thus, there is an induced filtration

$$
M(n p, m p-1) \otimes L(1,0)=\left[\begin{array}{l}
M(n p-1, m p) \\
M(n p, m p-2)
\end{array}\right.
$$

Let $N$ denote the quotient $(M(n p, m p-1) \otimes L(1,0)) / M(n p, m p-2)$. We have an exact sequence

$$
0 \rightarrow M(n p, m p-2) \rightarrow M(n p, m p-1) \otimes L(1,0) \rightarrow N \rightarrow 0 .
$$


The support variety of the middle term $M(n p, m p-1) \otimes L(1,0)$ is proper. Now, $N$ sits in an exact sequence:

$$
0 \rightarrow M(n p-1, m p) \rightarrow N \rightarrow M(n p+1, m p-1) \rightarrow 0 .
$$

The support variety of the last term in this sequence, $M(n p+1, m p-1)$, is proper by the $p \mid \lambda_{2}+1$ case.

Thus, if the support of $M(n p, m p-2)$ is proper, then the same holds for $N$ and hence, by the second sequence, the same holds for $M(n p-1, m p)$. On the other hand, if the support of $M(n p-1, m p)$ is proper, the second sequence implies that the same holds for $N$ and, thus, by the first sequence, the same holds for $M(n p, m p-2)$ (see [14, (2.2.7)] for properties of support varieties and exact sequences).

LEMMA 6.4. The support variety $\mathcal{V}_{B_{1}}(M(n p, m p-2))$ is proper for all $n \geq 0, m>0$ if $\mathcal{V}_{B_{1}}(M(n p, p-2))$ is proper for all $n \geq 0$.

Proof. The results follow by induction on $m$. Suppose that $\mathcal{V}_{B_{1}}(M(n p, k p-2))$ is proper for all $n \geq 0$ and all $0 \leq k \leq m$. We prove that $\mathcal{V}_{B_{1}}(M(n p,(m+1) p-2))$ is proper. Consider the tensor product $M(n p, m p-2) \otimes L(0,1)^{(1)}$. As in Lemma 6.3, we use the tensor identity and a filtration on $L(0,1)^{(1)}$. We have

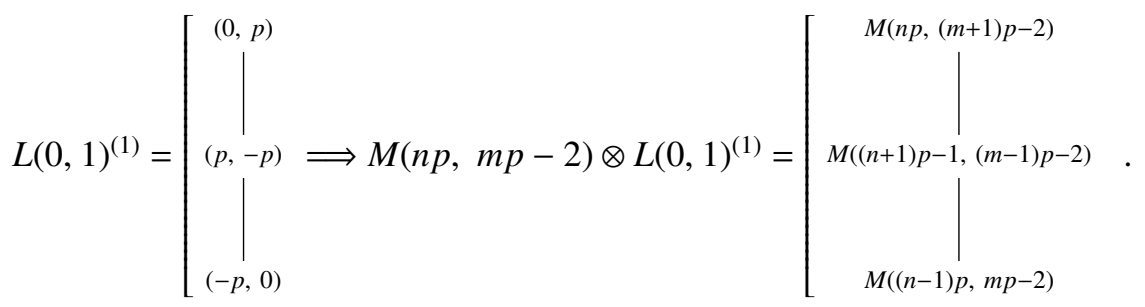

Let $N$ be the submodule such that

$$
\left(M(n p, m p-2) \otimes L(0,1)^{(1)}\right) / N \cong M(n p,(m+1) p-2) .
$$

The filtration on $N$ has subquotients whose supports are proper by the induction hypothesis, hence $N$ has proper support. It follows that $M(n p,(m+1) p-2)$ has proper support.

Finally, we prove that modules of the form $M(n p, p-2)$ have proper support. This will finish off the calculation for $l(w)=2$ when $\Phi=A_{2}$ with $p \geq 3$.

Lemma 6.5. The support variety $\mathcal{V}_{B_{1}}(M(n p, p-2))$ is proper. Hence,

$$
\mathcal{V}_{B_{1}}(M(n p, p-2))=\mathfrak{u}_{\{\alpha\}} \cup \mathfrak{u}_{\{\beta\}} .
$$

Proof. We argue by induction on $n$. The base case is $M(0, p-2)$. This module has proper support by Corollary 5.2, since $\Phi_{\lambda, p}=\{\alpha+\beta\}$. Assume that $M(k p, p-2)$ has proper support for all $0 \leq k \leq n$. We show that $M((n+1) p, p-2)$ has proper support. 
As in the previous two lemmas, we consider a tensor product, in this case $M(n p, p-2) \otimes L(1,0)^{(1)}$. The filtration on $(n p, p-2) \otimes L(1,0)^{(1)}$ now has socle the one-dimensional $B$-module $(n p,-2)$, which is not a dominant weight, so we are forced to consider the higher derived functors $R^{i} F, i>0$.

The $G$-module $L(1,0)^{(1)}$ has a $B$-filtration with sections of the form

$$
L(1,0)^{(1)}=\left[\begin{array}{c}
(p, 0) \\
\mid \\
(0,-p, p)
\end{array} .\right.
$$

Tensoring with $(n p, p-2)$ gives an exact sequence of $B$-modules:

$$
0 \rightarrow(n p,-2) \rightarrow L(1,0)^{(1)} \otimes(n p, p-2) \rightarrow\left[\left.\right|_{(-p, p)} ^{(p, 0)} \otimes(n p, p-2) \rightarrow 0 .\right.
$$

Applying the induction functor $F(\cdot)$, we have a long exact sequence in cohomology:

$$
\begin{aligned}
0 \longrightarrow F(n p,-2) & \longrightarrow L(1,0)^{(1)} \otimes F(n p, p-2) \longrightarrow F\left(\left[\left.\right|_{(-p, p)} ^{(p, 0)} \otimes(n p, p-2)\right)\right. \\
\longrightarrow & R^{1} F(n p,-2) \longrightarrow 0 .
\end{aligned}
$$

The first term, $F((n p,-2))$, vanishes since $(n p,-2)$ is not $\beta$-dominant, so (6.4) is a short exact sequence. Also note that the second term has proper support by the induction hypothesis. Now, we claim that the module $R^{1} F((n p,-2))$ has proper support.

Recall that $F(\cdot)=\operatorname{ind}_{B}^{P_{\langle\alpha\rangle}}\left(\operatorname{ind}_{B}^{P_{\{\beta\rangle}}(\cdot)\right)$. Consider the spectral sequence

$$
E_{2}^{i, j}=R^{i} \operatorname{ind}_{B}^{P_{\langle\alpha\rangle}} R^{j} \operatorname{ind}_{B}^{P_{\langle\beta\rangle}}(n p,-2) \Rightarrow R^{i+j} F(n p,-2) .
$$

Set $E_{1}=R^{1} F((n p,-2))$. The spectral sequence yields a five-term exact sequence of the form

$$
\begin{aligned}
0 \longrightarrow & R^{1} \operatorname{ind}_{B}^{P_{\langle\alpha\rangle}}\left(\operatorname{ind}_{B}^{P_{\langle\beta\rangle}}((n p,-2))\right) \longrightarrow E_{1} \\
& \longrightarrow \operatorname{ind}_{B}^{P_{\langle\alpha\rangle}}\left(R^{1} \operatorname{ind}_{B}^{P_{\langle\beta\rangle}}((n p,-2))\right) \longrightarrow R^{2} \operatorname{ind}_{B}^{P_{\langle\alpha\rangle}}\left(\operatorname{ind}_{B}^{P_{\langle\beta\rangle}}((n p,-2))\right) \longrightarrow \cdots
\end{aligned}
$$

Since $\operatorname{ind}_{B}^{P_{\langle\beta\rangle}}((n p,-2))=0$, the first and last terms vanish, so we have

$$
E_{1} \cong \operatorname{ind}_{B}^{P_{\langle\alpha\}}}\left(R^{1} \operatorname{ind}_{B}^{P_{\langle\beta\}}}((n p,-2))\right) \text {. }
$$


By Serre duality [12, Proposition II 5.2(c)],

$$
R^{1} \operatorname{ind}_{B}^{P_{\{\beta\}}}((n p,-2)) \cong(n p-1,0) .
$$

Consequently, from the $l\left(w^{\prime}\right)=1$ case, we can conclude that $E_{1} \cong \operatorname{ind}_{B}^{P_{\langle\alpha\rangle}}((n p-1,0))$ has proper support.

Now (6.4) implies that the module

$$
F\left(\int_{(-p, p)}^{(p, 0)} \otimes(n p, p-2)\right)
$$

has proper support. We have an exact sequence

$$
0 \longrightarrow F((n-1) p, 2 p-2) \longrightarrow F\left(\left[\left.\right|_{(-p, p)} ^{(p, 0)} \otimes(n p, p-2)\right) \longrightarrow F((n+1) p, p-2) \longrightarrow 0 .\right.
$$

The middle term has proper support and we need to show that the last term has proper support. Thus, it suffices to show that $F((n-1) p, 2 p-2)$ has proper support. This is the other base case in our double induction.

We argue as in Lemma 6.4 with $n$ replaced by $n-1$ and $m=1$. Now the $B$ filtration on $((n-1) p, p-2) \otimes L(0,1)^{(1)}$ has a nondominant weight in the middle layer $((n+1) p,-2)$. Let $N$ denote the quotient

$$
N:=\left(((n-1) p, p-2) \otimes L(0,1)^{(1)}\right) /((n-2) p, p-2),
$$

so that $N$ has socle consisting of $((n+1) p,-2)$. We have an exact sequence

$$
0 \longrightarrow F((n-2) p, p-2) \longrightarrow F\left[((n-1) p, p-2) \otimes L(0,1)^{(1)}\right] \longrightarrow F(N) \longrightarrow 0
$$

with first and middle terms having proper support; thus, $F(N)$ has proper support. Furthermore, $N$ sits in a sequence

$$
0 \longrightarrow((n+1) p,-2) \longrightarrow N \longrightarrow((n-1) p, 2 p-2) \longrightarrow 0
$$

and applying $F(\cdot)$ we have

$$
\begin{gathered}
0 \longrightarrow F((n+1) p,-2) \longrightarrow F(N) \longrightarrow F((n-1) p, 2 p-2) \\
\longrightarrow R^{1} F((n+1) p,-2) \longrightarrow R^{1} F(N) \longrightarrow 0 .
\end{gathered}
$$

The term $F((n+1) p,-2)$ vanishes and so does $R^{1} F(N)$ by extending the sequence (6.5). As before, we identify

$$
R^{1} F((n+1) p,-2) \cong \operatorname{ind}_{B}^{P_{\{\alpha\}}}((n+1) p-1,0),
$$

which has proper support. Thus, the term $F((n-1) p, 2 p-2)$ has proper support and the proof is concluded. 
TABle 4. $B_{1}$-support varieties for $A_{2}$ when $\ell(w) \neq 2, p=2$.

\begin{tabular}{ccl}
\hline$w$ & $\mathcal{V}_{B_{1}}\left(H^{0}(w, \lambda)\right)$ & $\lambda$ \\
\hline$e$ & $\mathfrak{H}_{\{\alpha\}} \cup \mathfrak{H}_{\{\beta\}}$ & All $\lambda$ \\
$s_{\alpha}$ & $\mathfrak{u}_{\{\alpha\}}$ & $p \mid \lambda_{1}+1$ \\
& $\mathfrak{H}_{\{\alpha\}} \cup \mathfrak{H}_{\{\beta\}}$ & $p \nmid \lambda_{1}+1$ \\
$s_{\beta}$ & $\mathfrak{H}_{\{\beta\}}$ & $p \mid \lambda_{2}+1$ \\
& $\mathfrak{u}_{\{\alpha\}} \cup \mathfrak{H}_{\{\beta\}}$ & $p \nmid \lambda_{2}+1$ \\
$s_{\alpha} s_{\beta} s_{\alpha}$ & $\mathcal{V}_{G_{1}}\left(H^{0}(\lambda)\right) \cap\left(\mathfrak{H}_{\{\alpha\}} \cup \mathfrak{H}_{\{\beta\}}\right)$ & All $\lambda$ \\
\hline
\end{tabular}

If $p=2$, then $\mathcal{N}_{1}(\mathfrak{u})=\mathfrak{u}_{\{\alpha\}} \cup \mathfrak{u}_{\{\beta\}}$. One can apply results from Sections 4 and 5 to give explicit descriptions of the $B_{1}$-supports of $H^{0}(w, \lambda)$ when $l(w) \neq 2$. We summarize the results in Table 4.

In the case where $l(w)=2$, it suffices (by symmetry) to consider $w=s_{\alpha} s_{\beta}$. We note that there are no $p$-regular weights and $\operatorname{dim} M(\lambda)$ is always divisible by 2 . Moreover, we do not need to show properness because all $B_{1}$-support varieties are already contained in $\mathfrak{u}_{\{\alpha\}} \cup \mathfrak{u}_{\{\beta\}}$. The following result summarizes the case where $l(w)=2$.

TheOREM 6.6. Let $w=s_{\alpha} s_{\beta}$. The $B_{1}$-support variety $\mathcal{V}=\mathcal{V}_{B_{1}}(M(\lambda))$ is given by

$$
\mathcal{V}_{B_{1}}(M(\lambda))= \begin{cases}\mathfrak{u}_{\{\alpha\}} & \text { if } \lambda=(2 n-1,0), \text { where } n \geq 1, \\ \mathfrak{u}_{\{\alpha\}} \cup \mathfrak{u}_{\{\beta\}} & \text { if } \lambda=(2 n, 0), \text { where } n \geq 0, \\ \mathfrak{u}_{\{\alpha\}} \cup \mathfrak{u}_{\{\beta\}} & \text { if } \lambda_{2} \neq 0 .\end{cases}
$$

\section{References}

[1] H. H. Andersen, 'Schubert varieties and Demazure's character formula', Invent. Math. 79 (1985), 611-618.

[2] C. P. Bendel, 'Support varieties for infinitesimal algebraic groups', PhD Thesis, Northwestern University, 1996.

[3] D. J. Benson, Representations and Cohomology I., Cambridge Studies in Advanced Mathematics, 30 (Cambridge University Press, Cambridge, 1991).

[4] A. Bjorner and F. Brenti, Combinatorics of Coxeter Groups, Graduate Texts in Mathematics, 231 (Springer, New York, 2005).

[5] N. Bourbaki, Groupes et Algèbres de Lie (Hermann, Paris, 1968).

[6] J. F. Carlson, Z. Lin, D. K. Nakano and B. J. Parshall, 'The restricted nullcone', Contemp. Math. 325 (2003), 51-75.

[7] E. Cline, B. Parshall and L. Scott, 'A Mackey imprimitivity theory for algebraic groups', Math. Z. 182 (1983), 447-471.

[8] E. M. Friedlander and B. J. Parshall, 'Support varieties for restricted Lie algebras', Invent. Math. 86 (1986), 553-562.

[9] E. M. Friedlander and J. Pevtsova, ' $\Pi$ supports for modules over finite group schemes', Duke Math. J. 139 (2007), 317-368.

[10] E. M. Friedlander and A. Suslin, 'Cohomology of finite group schemes over a field', Invent. Math. 127(2) (1997), 209-270. 
[11] J. E. Humphreys, Reflection Groups and Coxeter Groups (Cambridge University Press, Cambridge, 1994).

[12] J. C. Jantzen, Representations of Algebraic Groups (Academic Press, New York, 1987).

[13] A. Melnikov, 'On varieties in an orbital variety closure in semisimple Lie algebra', J. Algebra 295 (2006), 44-50.

[14] D. K. Nakano, B. J. Parshall and D. C. Vella, 'Support varieties for algebraic groups', J. reine angew. Math. 547 (2002), 15-49.

[15] P. Polo, 'Variétés de Schubert et excellentes filtrations', Astérisque 173-174 (1989), 10-11, 281-311.

[16] A. Suslin, E. M. Friedlander and C. P. Bendel, 'Infinitesimal 1-parameter subgroups and cohomology', J. Amer. Math. Soc. 10 (1997), 693-728.

[17] A. Suslin, E. M. Friedlander and C. P. Bendel, 'Support varieties for infinitesimal group schemes', J. Amer. Math. Soc. 10 (1997), 729-759.

[18] University of Georgia VIGRE Algebra Group, 'Support varieties for Weyl modules over bad primes', J. Algebra 312 (2007), 602-633.

[19] W. van der Kallen, 'Longest weight vectors and excellent filtrations', Math. Z. 201 (1989), 19-31.

BENJAMIN F. JONES, Department of Mathematics,

Statistics, and Computer Science, University of Wisconsin-Stout,

Menomonie, WI 54751, USA

e-mail: jonesbe@uwstout.edu

DANIEL K. NAKANO, Department of Mathematics, University of Georgia,

Athens, GA 30602, USA

e-mail: nakano@math.uga.edu 